QUANTUM PROBABILITY

BANACH CENTER PUBLICATIONS, VOLUME 73

INSTITUTE OF MATHEMATICS

POLISH ACADEMY OF SCIENCES

WARSZAWA 2006

\title{
DECOMPOSABILITY OF EXTREMAL POSITIVE UNITAL MAPS ON $M_{2}(\mathbb{C})$
}

\author{
WŁADYSŁAW A. MAJEWSKI and MARCIN MARCINIAK \\ Institute of Theoretical Physics and Astrophysics, Gdańsk University \\ Wita Stwosza 57, 80-952 Gdańsk, Poland \\ E-mail: fizwam@univ.gda.pl,matmm@univ.gda.pl
}

\begin{abstract}
A map $\varphi: M_{m}(\mathbb{C}) \rightarrow M_{n}(\mathbb{C})$ is decomposable if it is of the form $\varphi=\varphi_{1}+\varphi_{2}$ where $\varphi_{1}$ is a CP map while $\varphi_{2}$ is a co-CP map. It is known that if $m=n=2$ then every positive map is decomposable. Given an extremal unital positive map $\varphi: M_{2}(\mathbb{C}) \rightarrow M_{2}(\mathbb{C})$ we construct concrete maps (not necessarily unital) $\varphi_{1}$ and $\varphi_{2}$ which give a decomposition of $\varphi$. We also show that in most cases this decomposition is unique.
\end{abstract}

1. Introduction. If $\mathcal{A}$ is a $C^{*}$-algebra and $n \in \mathbb{N}$ then by $M_{n}(\mathcal{A})$ we denote the $C^{*}$ algebra of square $n \times n$-matrices with coefficients in $\mathcal{A}$. In particular $M_{n}(\mathbb{C})$ is the algebra of matrices with complex entries. For each $m, n \in \mathbb{N}$ we have the following isomorphisms:

$$
M_{m}\left(M_{n}(\mathbb{C})\right) \cong M_{m}(\mathbb{C}) \otimes M_{n}(\mathbb{C}) \cong M_{m n}(\mathbb{C}) .
$$

It follows that $M_{m}\left(M_{n}(\mathbb{C})\right)$ has the natural structure of a $C^{*}$-algebra. In particular one defines the conjugation of $\mathbf{A}=\left[A_{i j}\right]_{i, j=1}^{m} \in M_{m}\left(M_{n}(\mathbb{C})\right.$ ) (where $A_{i j} \in M_{n}(\mathbb{C})$ for $i, j=1, \ldots, m)$ by the formula $\mathbf{A}^{*}=\left[A_{j i}^{*}\right]_{i, j=1}^{m}$. Recall (see for example $[6,2]$ ) that A is positive in $M_{m}\left(M_{n}(\mathbb{C})\right)$ if and only if $\sum_{i, j=1}^{m} \overline{\mu_{i}} \mu_{j}\left\langle v_{i}, A_{i j} v_{j}\right\rangle \geq 0$ (i.e. the matrix $\left[\left\langle v_{i}, A_{i j} v_{j}\right\rangle\right]_{i, j=1}^{m}$ is a positive element of $\left.M_{m}(\mathbb{C})\right)$ for every $v_{1}, \ldots, v_{m} \in \mathbb{C}^{n}$ and $\mu_{1}, \ldots, \mu_{m} \in \mathbb{C}$. We say that $\mathbf{A}$ is block-positive if $\sum_{i, j=1}^{m} \overline{\mu_{i}} \mu_{j}\left\langle v, A_{i j} v\right\rangle \geq 0$ (i.e. the ma$\operatorname{trix}\left[\left\langle v, A_{i j} v\right\rangle\right]_{i, j=1}^{m}$ is positive in $\left.M_{m}(\mathbb{C})\right)$ for every $v \in \mathbb{C}^{n}$ and $\mu_{1}, \ldots, \mu_{m} \in \mathbb{C}$. For every $\mathbf{A}=\left[A_{i j}\right]_{i, j=1}^{m} \in M_{m}\left(M_{n}(\mathbb{C})\right)$ we define the partial transposition of $\mathbf{A}$ by $\mathbf{A}^{\tau}=\left[A_{j i}\right]_{i, j=1}^{m}$. Note the difference between this operation and the usual transposition $\mathbf{A} \mapsto \mathbf{A}^{T}$ on the

2000 Mathematics Subject Classification: 47B65, 47L07.

Key words and phrases: positive maps, decomposable maps, face structure.

W.A.M. was partially supported by the grant PBZ-MIN-008/PO3/2003, while M.M. was partially supported by KBN grant 2P04A00723. The authors would also like to thank for the support of EU RTN HPRN-CT-2002-00729 and Poland-South Africa Cooperation Joint project.

The paper is in final form and no version of it will be published elsewhere. 
algebra $M_{m n}(\mathbb{C})(\mathrm{cf} .(1.1))$ : the usual transposition preserves positivity of $\mathbf{A}$ while, for $n, m \geq 2$, partial transposition does not!

A linear map $\varphi: M_{m}(\mathbb{C}) \rightarrow M_{n}(\mathbb{C})$ is called a positive map if $\varphi(A)$ is a positive matrix for every positive matrix $A \in M_{m}(\mathbb{C})$. If $k \in \mathbb{N}$ then $\varphi$ is called $k$-positive map (respectively $k$-copositive map) whenever $\left[\varphi\left(A_{i j}\right)\right]_{i, j=1}^{k}$ (respectively $\left[\varphi\left(A_{j i}\right)\right]_{i, j=1}^{k}$ ) is a positive element in the algebra $M_{k}\left(M_{n}(\mathbb{C})\right)$ for every positive element $\left[A_{i j}\right]_{i, j=1}^{k}$ from $M_{k}\left(M_{m}(\mathbb{C})\right)$. If $\varphi$ is $k$-positive (respectively $k$-copositive) for every $k \in \mathbb{N}$ then $\varphi$ is called completely positive or CP (respectively completely copositive or co-CP). A positive map which is a sum of completely positive and completely copositive maps is called decomposable ${ }^{1}$.

Let $\left\{E_{i j}\right\}_{i, j=1}^{m}$ be a system of matrix units in $M_{m}(\mathbb{C})$ and $\mathbf{H}_{\varphi}=\left[\varphi\left(E_{i j}\right)\right]_{i, j=1}^{m} \in$ $M_{m}\left(M_{n}(\mathbb{C})\right)$ be Choi matrix of $\varphi$ with respect to the system $\left\{E_{i j}\right\}$ ([1], see also [4]). Recall the following

Theorem 1.1 ([1], see also [4]). Let $\varphi: M_{m}(\mathbb{C}) \rightarrow M_{n}(\mathbb{C})$ be a linear map. Then

1. the map $\varphi$ is positive if and only if the matrix $\mathbf{H}_{\varphi}$ is block-positive;

2. the map $\varphi$ is completely positive (respectively completely copositive) if and only if $\mathbf{H}_{\varphi}$ (respectively $\mathbf{H}_{\varphi}^{\tau}$ ) is a positive element of $M_{m}\left(M_{n}(\mathbb{C})\right)$.

We say that a positive map $\varphi$ is unital if $\varphi(\mathbb{I})=\mathbb{I}$ where $\mathbb{I}$ denotes the identity matrix of the respective algebra. The set of all positive (respectively completely positive, completely copositive, decomposable) maps from $M_{m}(\mathbb{C})$ into $M_{n}(\mathbb{C})$ will be denoted by $\mathcal{P}(m, n)$ (respectively $\mathcal{C} \mathcal{P}(m, n), \mathcal{C} c \mathcal{P}(m, n), \mathcal{D}(m, n))$. We will write simply $\mathcal{P}, \mathcal{C P}$, $\mathcal{C} c \mathcal{P}$ and $\mathcal{D}$ instead of $\mathcal{P}(m, n), \mathcal{C P}(m, n), \mathcal{C} c \mathcal{P}(m, n)$ and $\mathcal{D}(m, n)$ when no confusion can arise. Observe that all of these sets have the structure of a convex cone. By $\mathcal{P}_{1}, \mathcal{C} \mathcal{P}_{1}$, $\mathcal{C} c \mathcal{P}_{1}$ and $\mathcal{D}_{1}$ we will denote the subsets of unital maps from respective cones. All of them are convex subsets.

Let $C$ be a convex cone and $c \in C$. We say that $c$ is an extreme point of $C$ if for every $c_{1}, c_{2} \in C$ the equality $c=c_{1}+c_{2}$ implies $c_{1}=\lambda c$ and $c_{2}=(1-\lambda) c$ for some $0 \leq \lambda \leq 1$. The generalization of the notion of extremality leads to the face structure of the cone $C$. Namely, we say that a convex subcone $F \subset C$ is a face of $C$ if for every $c_{1}, c_{2} \in C$ the condition $c_{1}+c_{2} \in F$ implies $c_{1}, c_{2} \in F$. Kye in [3] gave an interesting characterization of maximal faces of the cone $\mathcal{P}(m, n)$

Theorem $1.2([3]) . A$ convex subset $F \subset \mathcal{P}(m, n)$ is a maximal face of $\mathcal{P}(m, n)$ if and only if there are vectors $\xi \in \mathbb{C}^{m}$ and $\eta \in \mathbb{C}^{n}$ such that $F=F_{\xi, \eta}$ where

$$
F_{\xi, \eta}=\left\{\varphi \in \mathcal{P}(m, n): \varphi\left(P_{\xi}\right) \eta=0\right\}
$$

and $P_{\xi}$ denotes the one-dimensional orthogonal projection in $M_{m}(\mathbb{C})$ onto the subspace generated by the vector $\xi$.

\footnotetext{
${ }^{1}$ In this paper we follow the definition of decomposability given by Størmer in [5]. Note that there is no connection of this notion with decomposable maps considered by U. Haagerup in the theory of operator spaces (see [2]).
} 
2. The case $m=n=2$. In this section we analyze in detail the case $m=n=2$. In [5] the following characterization of extremal points of $\mathcal{P}_{1}$ is given:

ThEOREM 2.1. A positive unital map $\varphi: M_{2}(\mathbb{C}) \rightarrow M_{2}(\mathbb{C})$ is an extremal point of $\mathcal{P}_{1}$ if and only if there are unitary operators $V, W \in U(2)$ such that the Choi matrix of the map $\varphi_{V, W}: A \mapsto V^{*} \varphi\left(W A W^{*}\right) V$ has the form

$$
\left[\begin{array}{cc|cc}
1 & 0 & 0 & y \\
0 & b & \bar{z} & t \\
\hline 0 & z & 0 & 0 \\
\bar{y} & \bar{t} & 0 & u
\end{array}\right]
$$

where the coefficients fulfil the following relations:

1. $b \geq 0, u \geq 0$ and $b+u=1$,

2. $|t|^{2}=2 b\left(u-|y|^{2}-|z|^{2}\right)$ in the case when $b \neq 0$, and $|y|=1$ or $|z|=1$ when $b=0$.

By $\left\{e_{1}, e_{2}\right\}$ we denote the canonical basis in $\mathbb{C}^{2}$. Let $\varphi$ be an extremal positive unital map as in Theorem 2.1. One can observe that $\varphi \in F_{\xi, \eta}$ for $\xi=W e_{2}$ and $\eta=V e_{1}$ where $V, W$ are the unitary operators from Theorem 2.1. Suppose that $\varphi=\varphi_{1}+\varphi_{2}$ where $\varphi_{1}$ is a completely positive map while $\varphi_{2}$ is a completely copositive one. Then both $\varphi_{1}$ and $\varphi_{2}$ should be elements of $F_{\xi, \eta}$ because $F_{\xi, \eta}$ is a face (cf. Theorem 1.2).

REMARK 2.2. There are extremal maps of the form (2.1) which are neither completely positive nor completely copositive (see Example 2.6 below). On the other hand theorem of Woronowicz (see [7]) asserts that every map from $\mathcal{P}(2,2)$ is decomposable. Hence, the maps $\varphi_{1}$ and $\varphi_{2}$ giving the decomposition of an extremal element of $\mathcal{P}_{1}(2,2)$ do not need be scalar multiples of unital maps.

In the sequel we will use the following lemmas.

Lemma 2.3. Let $\psi \in F_{\xi, \eta}$ for some $\xi, \eta \in \mathbb{C}^{2}$, and $V$ and $W$ be unitary operators from $M_{2}(\mathbb{C})$ such that $\xi=W e_{2}$ and $\eta=V e_{1}$. Then the Choi matrix of the map $\psi_{V, W}$ has the form

$$
\left[\begin{array}{ll|ll}
a & c & 0 & y \\
\bar{c} & b & \bar{z} & t \\
\hline 0 & z & 0 & 0 \\
\bar{y} & \bar{t} & 0 & u
\end{array}\right]
$$

for some $a, b, u \geq 0$ and $c, y, z, t \in \mathbb{C}$. Moreover, the following conditions hold:

1. $|c|^{2} \leq a b$,

2. $|t|^{2} \leq b u$,

3. $(|y|+|z|)^{2} \leq a u$.

Proof. Let us write briefly $\psi^{\prime}$ instead of $\psi_{V, W}$. The Choi matrix of the map $\psi^{\prime}$ has the form

$$
\mathbf{H}=\left[\begin{array}{ll}
\psi^{\prime}\left(E_{11}\right) & \psi^{\prime}\left(E_{12}\right) \\
\psi^{\prime}\left(E_{21}\right) & \psi^{\prime}\left(E_{22}\right)
\end{array}\right] .
$$


From the condition $(1.2)$ we get

$$
\psi^{\prime}\left(E_{22}\right) e_{1}=V^{*} \psi\left(W E_{22} W^{*}\right) V e_{1}=V^{*} \psi\left(P_{\xi}\right) \eta=0 .
$$

Because $\psi^{\prime}\left(E_{22}\right)$ is a positive element of $M_{2}(\mathbb{C})$ we have $\psi^{\prime}\left(E_{22}\right)=u E_{22}$ for some $u \geq 0$. Hence $\psi^{\prime}\left(E_{22}\right)=\left[\begin{array}{ll}0 & 0 \\ 0 & u\end{array}\right]$. Positivity of $\psi^{\prime}$ implies also that $\psi^{\prime}\left(E_{11}\right)$ is a hermitian matrix of the general form $\psi^{\prime}\left(E_{11}\right)=\left[\begin{array}{ll}a & c \\ \bar{c} & b\end{array}\right]$ with $a, b \geq 0$ and $\operatorname{det} \psi^{\prime}\left(E_{11}\right)=a b-|c|^{2} \geq 0$, so we have (1). Let $\psi^{\prime}\left(E_{12}\right)=\left[\begin{array}{ll}x & y \\ \bar{z} & t\end{array}\right]$ for some $x, y, z, t \in \mathbb{C}$. Because $\psi^{\prime}$ is positive we have $\psi^{\prime}\left(E_{21}\right)=\psi^{\prime}\left(E_{12}^{*}\right)=\psi^{\prime}\left(E_{12}\right)^{*}=\left[\begin{array}{ll}\bar{x} & z \\ \bar{y} & \bar{t}\end{array}\right]$. By Theorem $1.1 \mathbf{H}$ is block-positive, hence the matrix

$$
\left[\begin{array}{cc}
\left\langle e_{1}, \psi^{\prime}\left(E_{11}\right) e_{1}\right\rangle & \left\langle e_{1}, \psi^{\prime}\left(E_{12}\right) e_{1}\right\rangle \\
\left\langle e_{1}, \psi^{\prime}\left(E_{21}\right) e_{1}\right\rangle & \left\langle e_{1}, \psi^{\prime}\left(E_{22}\right) e_{1}\right\rangle
\end{array}\right]=\left[\begin{array}{cc}
a & x \\
\bar{x} & 0
\end{array}\right]
$$

is positive and consequently $x=0$. Thus we arrived at the form (2.2). Another application of block-positivity of $H$ leads to the conclusion that the matrix

$$
\left[\begin{array}{cc}
\left\langle e_{2}, \psi^{\prime}\left(E_{11}\right) e_{2}\right\rangle & \left\langle e_{2}, \psi^{\prime}\left(E_{12}\right) e_{2}\right\rangle \\
\left\langle e_{2}, \psi^{\prime}\left(E_{21}\right) e_{2}\right\rangle & \left\langle e_{2}, \psi^{\prime}\left(E_{22}\right) e_{2}\right\rangle
\end{array}\right]=\left[\begin{array}{cc}
b & t \\
\bar{t} & u
\end{array}\right]
$$

is positive, hence we get inequality (2).

Let $\omega$ be a linear functional on $M_{2}(\mathbb{C})$. By Corollary 8.4 in [5] $\omega$ is a positive functional if and only if $\omega\left(E_{11}\right) \geq 0, \omega\left(E_{22}\right) \geq 0, \omega\left(E_{21}\right)=\overline{\omega\left(E_{12}\right)}$ and $\left|\omega\left(E_{12}\right)\right|^{2} \leq \omega\left(E_{11}\right) \omega\left(E_{22}\right)$. Let us denote $\alpha=\omega\left(E_{11}\right), \beta=\omega\left(E_{22}\right)$ and $\gamma=\omega\left(E_{12}\right)$. Obviously $\omega \circ \psi^{\prime}$ is a positive functional for every positive $\omega$. From another application of this result of Størmer we get that the inequality

$$
|\gamma y+\overline{\gamma z}+\beta t|^{2} \leq \beta u(\alpha a+\beta b+2 \Re(\gamma c))
$$

holds whenever $\alpha \geq 0, \beta \geq 0$ and $|\gamma|^{2} \leq \alpha \beta$. Inequality (2.3) can be written in the form

$$
|\gamma y+\overline{\gamma z}|^{2}+\beta^{2}|t|^{2}+2 \Re[(\gamma y+\overline{\gamma z}) \beta \bar{t}] \leq \beta u(\alpha a+\beta b+2 \Re(\gamma c)) .
$$

Putting here $-\gamma$ instead of $\gamma$ we get

$$
|\gamma y+\overline{\gamma z}|^{2}+\beta^{2}|t|^{2}-2 \Re[(\gamma y+\overline{\gamma z}) \beta \bar{t}] \leq \beta u(\alpha a+\beta b-2 \Re(\gamma c)) .
$$

If we add (2.4) and (2.5) and divide the result by 2 , then we get

$$
|\gamma y+\overline{\gamma z}|^{2}+\beta^{2}|t|^{2} \leq \beta u(\alpha a+\beta b) .
$$

This can be rewritten equivalently as

$$
|\gamma|^{2}\left(|y|^{2}+|z|^{2}\right)+2 \Re\left(y z \gamma^{2}\right)+\beta^{2}|t|^{2} \leq \beta u(\alpha a+\beta b)
$$

Let $\varepsilon>0$ and take $\alpha=\varepsilon^{-1}, \beta=\varepsilon$ and $\gamma$ such that $|\gamma|=1$ and $y z \gamma^{2}=|y||z|$. Then (2.7) has the form

$$
(|y|+|z|)^{2} \leq a u+\varepsilon^{2}\left(b u-|t|^{2}\right)
$$

Because $\varepsilon$ can be chosen arbitrarily small, we get the inequality (3) and the proof is finished. 
LEMMA 2.4. A map $\psi \in F_{\xi, \eta}$ is completely positive if and only if the coefficients of the matrix $\mathbf{H}$ from (2.2) fulfil the following conditions:

(A1) $z=0$,

(A2) $|y|^{2} \leq a u$

(A3) $|t|^{2} \leq b u$

(A4) $|c|^{2} \leq a b$

(A5) $a|t|^{2}+u|c|^{2} \leq b\left(a u-|y|^{2}\right)+2 \Re(c t \bar{y})$.

Analogously, $\psi$ is completely copositive if and only if the following conditions hold:

(B1) $y=0$,

(B2) $|z|^{2} \leq a u$,

(B3) $|t|^{2} \leq b u$,

(B4) $|c|^{2} \leq a b$,

(B5) $a|t|^{2}+u|c|^{2} \leq b\left(a u-|z|^{2}\right)+2 \Re(c \bar{t} \bar{z})$.

Proof. By Theorem 1.1 and properties of unitary equivalence $\psi$ is completely positive if and only if the matrix $\mathbf{H}$ is positive. This is equivalent to the fact that all principal minors of $\mathbf{H}$ are nonnegative. Conditions (A2), (A3) and (A4) follow from the fact that $\left|\begin{array}{ll}a & y \\ \bar{y} & u\end{array}\right| \geq 0,\left|\begin{array}{ll}b & t \\ \bar{t} & u\end{array}\right| \geq 0$ and $\left|\begin{array}{ll}a & c \\ \bar{c} & b\end{array}\right| \geq 0$. (A1) is a consequence of the equality $\operatorname{det} \mathbf{H}=$ $-|z|^{2}\left(a u-|y|^{2}\right)$ and (A2). Inequality in (A5) is equivalent to $\left|\begin{array}{lll}a & c & y \\ \bar{c} & b & t \\ \bar{y} & \bar{t} & u\end{array}\right| \geq 0$.

The second part of the lemma follows in the similar way from positivity of the matrix $\mathbf{H}^{\tau}$ in the case when $\psi^{\prime}$ is completely copositive.

REMARK 2.5. If $\varphi$ is an extremal positive unital map described in Theorem 2.1 with the Choi matrix (2.1) then by condition (3) from Lemma $2.3|y|+|z| \leq u^{1 / 2}$. Lemma 8.11 in [5] claims that in the case $b>0$ a stronger condition holds. Namely,

$$
|y|+|z|=u^{1 / 2} \text {. }
$$

Moreover, it follows from Lemma 8.8 in [5] that in this case

$$
t^{2}=-4(1-u) y \bar{z}
$$

Example 2.6. Consider the map $\psi: M_{2}(\mathbb{C}) \rightarrow M_{2}(\mathbb{C})$ with the Choi matrix

$$
\left[\begin{array}{cc|cc}
1 & 0 & 0 & \frac{1}{2} s \\
0 & 1-s^{2} & \frac{1}{2} s & i s\left(1-s^{2}\right)^{1 / 2} \\
\hline 0 & \frac{1}{2} s & 0 & 0 \\
\frac{1}{2} s & -i s\left(1-s^{2}\right)^{1 / 2} & 0 & s^{2}
\end{array}\right]
$$

where $0<s<1$. It follows from Theorem 2.1 (compare also with (2.9) and (2.10)) and Lemma 2.4 that $\psi$ is an extremal positive unital map which is neither completely positive nor completely copositive.

Now, we are ready to formulate our main theorem. 
THEOREM 2.7. Assume that $\varphi$ is an extremal positive unital map with the Choi matrix given in (2.1) and $u>0, y \neq 0, z \neq 0$. Then there are $\varphi_{1}, \varphi_{2} \in F_{\xi, \eta}$ such that $\varphi_{1}$ is completely positive, $\varphi_{2}$ is completely copositive and $\varphi=\varphi_{1}+\varphi_{2}$. Moreover, the pair $\varphi_{1}, \varphi_{2}$ is uniquely determined, and Choi matrices $\mathbf{H}_{1}, \mathbf{H}_{2}$ of maps $\varphi_{1}, \varphi_{2}$ have the following form:

$$
\begin{aligned}
\mathbf{H}_{1} & =\left[\begin{array}{cc|cc}
|y| u^{-1 / 2} & c & 0 & y \\
\bar{c} & |z|(1-u) u^{-1 / 2} & 0 & \frac{1}{2} t \\
\hline 0 & 0 & 0 & 0 \\
\bar{y} & \frac{1}{2} \bar{t} & 0 & |y| u^{1 / 2}
\end{array}\right], \\
\mathbf{H}_{2} & =\left[\begin{array}{cc|cc}
|z| u^{-1 / 2} & -c & 0 & 0 \\
-\bar{c} & |y|(1-u) u^{-1 / 2} & \bar{z} & \frac{1}{2} t \\
\hline 0 & z & 0 & 0 \\
0 & \frac{1}{2} \bar{t} & 0 & |z| u^{1 / 2}
\end{array}\right]
\end{aligned}
$$

where $u, y, z, t$ are the coefficients of the matrix (2.1) and $c$ is a complex number such that $c^{2}=-(1-u) u^{-1} y z$.

REMARK 2.8. The uniqueness of the decomposition of $\varphi$ onto $\varphi_{1}$ and $\varphi_{2}$ does not hold if the assumptions of the above theorem are not fulfilled. To see this let us consider the following cases:

1. $u=0$. Then the Choi matrix of the map $\varphi$ has the following form

$$
\mathbf{H}=\left[\begin{array}{ll|ll}
1 & 0 & 0 & 0 \\
0 & 1 & 0 & 0 \\
\hline 0 & 0 & 0 & 0 \\
0 & 0 & 0 & 0
\end{array}\right]
$$

and $\varphi$ is completely positive and completely copositive.

2. $y=0$. The Choi matrix of $\varphi$ has the form

$$
\mathbf{H}=\left[\begin{array}{cc|cc}
1 & 0 & 0 & 0 \\
0 & 1-|z|^{2} & \bar{z} & 0 \\
\hline 0 & z & 0 & 0 \\
0 & 0 & 0 & |z|^{2}
\end{array}\right]
$$

and the map $\varphi$ is completely copositive.

3. $z=0$. The Choi matrix of $\varphi$ has the form

$$
\mathbf{H}=\left[\begin{array}{cc|cc}
1 & 0 & 0 & y \\
0 & 1-|y|^{2} & 0 & 0 \\
\hline 0 & 0 & 0 & 0 \\
\bar{y} & 0 & 0 & |y|^{2}
\end{array}\right]
$$

and the map $\varphi$ is completely positive. 
Turning to non-uniqueness of the decomposition of $\varphi$, let $\varepsilon>0$ and put

$$
\mathbf{A}_{\varepsilon}=\left[\begin{array}{cc|cc}
0 & 0 & 0 & 0 \\
0 & \varepsilon & 0 & 0 \\
\hline 0 & 0 & 0 & 0 \\
0 & 0 & 0 & 0
\end{array}\right]
$$

Then, it follows from Lemma 2.4 that $\mathbf{A}_{\varepsilon}$ determines the map $\psi_{\varepsilon}$ which is completely positive and completely copositive. Moreover, in each of the above three cases the equality $\mathbf{H}=\left(\mathbf{H}-\mathbf{A}_{\varepsilon}\right)+\mathbf{A}_{\varepsilon}$ describes the decomposition onto $\mathrm{CP}$ and co-CP parts for every sufficiently small $\varepsilon$.

Proof of Theorem 2.7. The existence of the decomposition follows from $[4,5]$ and the discussion after Theorem 2.1. To show that the decomposition is unique (and has the required form) assume that it is given by $\mathbf{H}=\mathbf{H}_{1}+\mathbf{H}_{2}$, where

$$
\mathbf{H}_{1}=\left[\begin{array}{cc|cc}
a_{1} & c & 0 & y \\
\bar{c} & b_{1} & 0 & t_{1} \\
\hline 0 & 0 & 0 & 0 \\
\bar{y} & \overline{t_{1}} & 0 & u_{1}
\end{array}\right], \quad \mathbf{H}_{2}=\left[\begin{array}{cc|cc}
a_{2} & -c & 0 & 0 \\
-\bar{c} & b_{2} & \bar{z} & t_{2} \\
\hline 0 & z & 0 & 0 \\
0 & \overline{t_{2}} & 0 & u_{2}
\end{array}\right] .
$$

Then the above coefficients fulfil the following set of relations:

$$
\begin{gathered}
a_{1}+a_{2}=1, \\
b_{1}+b_{2}=1-u, \\
t_{1}+t_{2}=t, \\
u_{1}+u_{2}=u, \\
|y|^{2} \leq a_{1} u_{1}, \\
\left|t_{1}\right|^{2} \leq b_{1} u_{1}, \\
|c|^{2} \leq a_{1} b_{1}, \\
a_{1}\left|t_{1}\right|^{2}+u_{1}|c|^{2} \leq b_{1}\left(a_{1} u_{1}-|y|^{2}\right)+2 \Re\left(c t_{1} \bar{y}\right), \\
|z|^{2} \leq a_{2} u_{2}, \\
\left|t_{2}\right|^{2} \leq b_{2} u_{2}, \\
|c|^{2} \leq a_{2} b_{2}, \\
a_{2}\left|t_{2}\right|^{2}+u_{2}|c|^{2} \leq b_{2}\left(a_{2} u_{2}-|z|^{2}\right)-2 \Re\left(c \overline{t_{2}} \bar{z}\right) .
\end{gathered}
$$

We divide the rest of the proof into some lemmas. 
LEMma 2.9. The coefficients $a_{1}, a_{2}, u_{1}, u_{2}$ have the following form

$$
\begin{aligned}
& a_{1}=|y| u^{-1 / 2}, \\
& a_{2}=|z| u^{-1 / 2}, \\
& u_{1}=|y| u^{1 / 2}, \\
& u_{2}=|z| u^{1 / 2} .
\end{aligned}
$$

Proof. From (2.9) we have $|y| u^{-1 / 2}+|z| u^{-1 / 2}=1$. Let $p=|y| u^{-1 / 2}, q=a_{1}$ and $r=u_{1} u^{-1}$. Then $(2.18)$ gives

$$
p^{2} \leq q r
$$

while (2.9), (2.22), (2.14) and (2.17) lead to

$$
(1-p)^{2} \leq(1-q)(1-r)
$$

The system of inequalities (2.30) and (2.31) is equivalent to

$$
\frac{p^{2}}{q} \leq r \leq 1-\frac{(1-p)^{2}}{1-q}
$$

By simple calculations one can show that the inequality between the first and the last terms in (2.32) is equivalent to $(q-p)^{2} \leq 0$, so $q=p$. So, putting $p$ instead of $q$ in $(2.32)$ we obtain also $r=p$. Hence, we have

$$
\begin{aligned}
& a_{1}=q=p=|y| u^{-1 / 2} \\
& a_{2}=1-a_{1}=1-|y| u^{-1 / 2}=|z| u^{-1 / 2}, \\
& u_{1}=r u=p u=|y| u^{1 / 2} \\
& u_{2}=u-u_{1}=\left(u^{1 / 2}-|y|\right) u^{1 / 2}=|z| u^{1 / 2} .
\end{aligned}
$$

LEMMA 2.10. The following relations hold:

$$
\begin{gathered}
|y| t_{1}=y \bar{c} u^{1 / 2} \\
|z| t_{2}=-\bar{z} c u^{1 / 2} .
\end{gathered}
$$

Proof. Observe that application of (2.26)-(2.29) reduces inequalities (2.21) and (2.25) to

$$
|y| u^{-1 / 2}\left|t_{1}\right|^{2}+|y| u^{1 / 2}|c|^{2}-2 \Re\left(c t_{1} \bar{y}\right) \leq 0
$$

and

$$
|z| u^{-1 / 2}\left|t_{2}\right|^{2}+|z| u^{1 / 2}|c|^{2}+2 \Re\left(c \overline{t_{2}} \bar{z}\right) \leq 0
$$

respectively. Let $y_{1}, z_{1} \in \mathbb{C}$ be such that $y_{1}^{2}=y$ and $z_{1}^{2}=z$. Then $(2.35)$ and $(2.36)$ can be rewritten in the form

$$
\left|\overline{y_{1}} u^{-1 / 4} t_{1}-y_{1} u^{1 / 4} \bar{c}\right|^{2} \leq 0
$$

and

$$
\left|\overline{z_{1}} u^{-1 / 4} \overline{t_{2}}+z_{1} u^{1 / 4} \bar{c}\right|^{2} \leq 0 .
$$

These inequalities are equivalent to $\overline{y_{1}} u^{-1 / 4} t_{1}=y_{1} u^{1 / 4} \bar{c}$ and $\overline{z_{1}} u^{-1 / 4} \overline{t_{2}}=-z_{1} u^{1 / 4} \bar{c}$. Multiplication of both sides of the first equality by $y_{1} u^{1 / 4}$ leads to (2.33) while multiplication of the second one by $z_{1} u^{1 / 4}$ gives $(2.34)$. 
Corollary 2.11. $\left|t_{1}\right|=\left|t_{2}\right| \geq \frac{1}{2}|t|$.

Proof. The equality follows from (2.33) and (2.34) while the inequality is a consequence of (2.16) and the triangle inequality.

LEMMA 2.12. The following relations hold:

$$
\begin{aligned}
& b_{1}=|z|(1-u) u^{-1 / 2}, \\
& b_{2}=|y|(1-u) u^{-1 / 2} .
\end{aligned}
$$

Proof. From (2.10), (2.19), (2.23), (2.28), (2.29) and Corollary 2.11 we have the following inequalities

$$
(1-u)|y||z|=\frac{1}{4}|t|^{2} \leq\left|t_{1}\right|^{2} \leq b_{1} u_{1}=|y| u^{1 / 2} b_{1}
$$

and

$$
(1-u)|y||z|=\frac{1}{4}|t|^{2} \leq\left|t_{2}\right|^{2} \leq b_{2} u_{2}=|z| u^{1 / 2} b_{2} .
$$

From the first inequality we obtain

$$
b_{1} \geq|z|(1-u) u^{-1 / 2}
$$

while from the second one and (2.15) we have

$$
\begin{aligned}
b_{1} & =1-u-b_{2} \leq 1-u-|y|(1-u) u^{-1 / 2}= \\
& =\left(u^{1 / 2}-|y|\right)(1-u) u^{-1 / 2}=|z|(1-u) u^{-1 / 2} .
\end{aligned}
$$

Thus we obtain (2.37). In a similar way we get (2.38).

LEMMA 2.13. $\left|t_{1}\right|=\left|t_{2}\right|=\frac{1}{2}|t|$.

Proof. It follows from (2.19), Lemma 2.12, (2.28) and (2.10) that

$$
\left|t_{1}\right|^{2} \leq b_{1} u_{1}=|z|(1-u) u^{-1 / 2} \cdot|y| u^{1 / 2}=(1-u)|y||z|=\frac{1}{4}|t|^{2} .
$$

The converse inequality is included in Corollary 2.11.

Corollary 2.14. $t_{1}=t_{2}=\frac{1}{2} t$.

Proof. It easily follows from (2.16) and Lemma 2.13.

LEMMA 2.15. $c^{2}=-(1-u) u^{-1} y z$.

Proof. From (2.34) and Corollary 2.14 we obtain $c=-\frac{1}{2} z|z|^{-1} t u^{-1 / 2}$. Thus, (2.10) implies $c^{2}=-z^{2}|z|^{-2} \cdot(1-u) y \bar{z} u^{-1}=-(1-u) u^{-1} y z$.

The coefficient $c$ in (2.11) and (2.12) is uniquely determined. It can be described in the following way. Let $y_{1}, z_{1} \in \mathbb{C}$ be such that $y_{1}^{2}=y, z_{1}^{2}=z$ and $t=2 i(1-u)^{1 / 2} y_{1} \overline{z_{1}}$ (cf. (2.10)). The numbers $y_{1}, z_{1}$ are not uniquely determined by these conditions but the expression $y_{1} z_{1}$ is. Then, by $(2.34)$

$$
c=-i(1-u)^{1 / 2} u^{-1 / 2} y_{1} z_{1} .
$$

Combining the results of Lemmas 2.9, 2.12, 2.15 and Corollary 2.10 we end the proof of Theorem 2.7. 
COROLlary 2.16. If $\varphi$ is extremal positive unital map with the Choi matrix of the form (2.1) and assumptions of Theorem 2.7 are fulfilled then $\varphi(A)=U_{1} A U_{1}^{*}+U_{2} A^{T} U_{2}^{*}$ for every $A \in M_{2}(\mathbb{C})$, where $U_{1}, U_{2} \in M_{2}(\mathbb{C})$ are of the form

$$
U_{1}=\left[\begin{array}{cc}
y_{1} u^{-1 / 4} & 0 \\
i \overline{z_{1}}(1-u)^{1 / 2} u^{-1 / 4} & \overline{y_{1}} u^{1 / 4}
\end{array}\right], \quad U_{2}=\left[\begin{array}{cc}
z_{1} u^{-1 / 4} & 0 \\
-i \overline{y_{1}}(1-u)^{1 / 2} u^{-1 / 4} & \overline{z_{1}} u^{1 / 4}
\end{array}\right],
$$

where $y_{1}$ and $z_{1}$ are as in the proof of Theorem 2.7.

Acknowledgments. Part of the work was done during a visit of the authors at University of South Africa in Pretoria. The authors want to thank Louis E. Labuschagne for his kind hospitality and fruitful discussions.

\section{References}

[1] M.-D. Choi, Completely positive maps on complex matrices, Lin. Alg. Appl. 10 (1975), 285-290.

[2] E. G. Effros and Z.-J. Ruan, Operator Spaces, Clarendon Press, Oxford, 2000.

[3] S.-H. Kye, Facial structures for the positive linear maps between matrix algebras, Canad. Math. Bull. 39 (1) (1996), 74-82.

[4] W. A. Majewski and M. Marciniak, On a characterization of positive maps, J. Phys. A: Math. Gen. 34 (2001), 5863-5874.

[5] E. Størmer, Positive linear maps of operator algebras, Acta Math. 110 (1963), 233-278.

[6] M. Takesaki, Theory of Operator Algebras I, Springer-Verlag, Berlin, 2002.

[7] S. L. Woronowicz, Positive maps of low dimensional matrix algebras, Rep. Math. Phys. 10 (1976), 165-183. 